\title{
TRATAMENTO DAS INFECÇÕES EM PACIENTES COM PANCI- TOPENIA SECUNDÁRIA A TRATAMENTO CITORREDUTOR
}

\author{
TREATMENT OF INFECTIONS IN PATIENTS WITH PANCYTOPENIA \\ RESULTING FROM CYTOREDUCTIVE TREATMENT
}

Danielle Leão C. F. Souzaํ \& Eduardo Magalhães Rego²

\begin{abstract}
${ }^{1}$ Mestranda. ${ }^{2}$ Docente. Disciplina de Hematologia. Departamento de Clínica Médica. Faculdade de Medicina de Ribeirão Preto - USP. Correspondência: E. M. Rego. Laboratório de Hematologia. Hospital das Clínicas da Faculdade de Medicina de Ribeirão Preto. Av. Bandeirantes, 3900. CEP 14049-900 Ribeirão Preto - SP, Brasil. E-mail: emrego@hcrp.fmrp.usp.br
\end{abstract}

SOUZA DLCF \& REGO EM. Tratamento das infecções em pacientes com pancitopenia secundária a tratamento citorredutor. Medicina, Ribeirão Preto, 36: 446-452, abr./dez. 2003.

RESUMO - Febre de etiologia desconhecida é a causa mais freqüente de internação hospitalar de pacientes neutropênicos em quimioterapia. O paciente é, em geral, oligossintomático, e a avaliação inicial deve incluir história e exame físico completos e minuciosos, além de exames laboratoriais, procurando identificar a etiologia da febre e complicações decorrentes da quimioterapia. O tratamento com antibióticos de largo espectro deve ser iniciado prontamente, e o esquema terapêutico reavaliado $72 \mathrm{~h}$, depois, com base nos estudos microbiológicos. No texto, discorremos sobre o diagnóstico da neutropenia febril, suas particularidades e o manejo do paciente.

UNITERMOS - Neutropenia; diagnóstico. Antibióticos. Febre. Pancitopenia.

\section{1- INTRODUÇÃO}

A causa mais freqüente de complicações e morte relacionada ao tratamento de pacientes em quimioterapia é a infecção, e o fator de risco mais importante para infecção, em tais pacientes, é a neutropenia. A importância da neutropenia é demonstrada pela correlação direta, observada entre o grau, sua duração e a frequiência de infecções.

Ademais, o paciente hematológico apresenta, com frequência, outras causas de diminuição da defesa contra infecções, como redução da imunidade humoral ou celular (disfunção fagocitária, redução de gamaglobulinas), e aumento das condições que favorecem contato ou inoculação de diversos patógenos, como a quebra da barreira cutânea por dispositivos endovenosos ou infiltração tumoral, mucosite decorrente do tratamento, redução de reflexos protetores, como a tosse, e alteração da microbiota normal.

\section{2- DEFINIÇÃO}

Neutropenia febril pode ser definida como febre (temperatura igual ou maior que $38^{\circ} \mathrm{C}$ por, pelo menos, $1 \mathrm{~h}$ ou temperatura acima de $38,3^{\circ} \mathrm{C}$ a qualquer momento) detectada em paciente com menos de 500 neutrófilos $/ \mathrm{mm}^{3}$ ou com menos de 1.000 neutrófi$\mathrm{los} / \mathrm{mm}^{3}$, com tendência à redução deste número. $\mathrm{O}$ número dos neutrófilos, a velocidade de desenvolvimento da neutropenia e sua duração são importantes fatores determinantes do risco de infecção. 


\section{3- AVALIAÇÃo do PACIENTE}

Devido à baixa contagem de neutrófilos, os processos inflamatórios são frustros ou discretos, e, geralmente, há uma pobreza de sintomas ou sinais. Cerca de 48 a $60 \%$ dos pacientes neutropênicos com febre têm infecção oculta ou detectável.

Deve ser obtida história detalhada dos eventos recentes, hora de início da febre, sintomas de qualquer natureza, procedimentos invasivos, local da infusão da quimioterapia. A data e o tipo de quimioterapia são importantes para prever a duração da neutropenia e identificar fatores de risco para infecção, como a mucosite.

A dor, mesmo que pouco intensa, deve ser valorizada. O paciente deve ser examinado detalhadamente, em busca do provável foco. Os principais sítios de infecção são: pulmão, pele (incluindo sítios de venóclise e região periungueal), foco urinário, região perineal e perianal, faringe, região periodontal, esôfago e olhos.

Devem ser colhidos, antes do início de antibioticoterapia, culturas para bactérias e fungos: hemocultura (2 amostras de sangue periférico ou, se o paciente for portador de cateter venoso central, 1 amostra de sangue periférico e outra de sangue do cateter); urocultura; swabs nasal, anal e de orofaringe (somente à admissão, para caracterizar colonização por germes resistentes a oxacilina ou fungos invasivos, como Candida tropicalis, Aspergillus), e culturas de outros locais suspeitos, se houver, como de secreção pericateter ou coprocultura. A análise comparativa entre o número de colônias bacterianas, em amostras de sangue periférico e de cateter, é importante para determinar se o sítio da infecção é o cateter. Indica infecção relacionada ao cateter a obtenção do mesmo germe em uma cultura de sangue periférico e em número maior ou igual a $15 \mathrm{CFU}$, na cultura semiquantitativa da ponta do cateter. Caso o paciente apresente lesões orais, deve ser colhido exame citológico (esfregaço) para pesquisa de hifas de fungos ou de inclusões virais (coloração de Schorr).

Além de hemograma completo, devem ser feitos radiografias de tórax, exame de urina rotina, bem como avaliação laboratorial da função renal (uréia e creatinina) e hepática (TP, albumina, proteína total, bilirrubinas, TGO, TGP, $\gamma$ GT, FA, DHL).

Pode-se, mesmo em vigência de infecção nos mencionados sítios, não serem encontrados leucócitos em urina, escarro e outros líquidos. Tomografia computadorizada (CT) de alta resolução revela pneu- monia em mais da metade dos pacientes neutropênicos, com achados normais à radiografia de tórax.

\section{4- ANTIBIOTICOTERAPIA EMPÍRICA}

Como a progressão de infecções em neutropênicos pode ser muito rápida e fatal, e considerando-se que, à admissão, não é possível distinguir, com segurança, os pacientes nos quais a febre não é causada por infecção, a antibioticoterapia empírica é preconizada. Pacientes neutropênicos sem febre, mas com sinais de infecção devem ser tratados da mesma forma que os neutropênicos febris.

Na seleção do antibiótico, deve ser considerado o perfil de infecções do serviço, a freqüência dos tipos de bactéria e sua susceptibilidade a determinados antibióticos.

Mais de $80 \%$ das infecções, em pacientes imunocomprometidos, são adquiridas da microbiota colonizadora do próprio paciente, que é rapidamente substituída pela microbiota hospitalar, após a internação.

Em geral, as bactérias Gram-positivas são responsáveis por 60 a $70 \%$ das infecções documentadas, embora a taxa de Gram-negativas venha aumentando (Pseudomonas aeruginosa, Escherichia coli e espécies de Klebsiella). No Brasil, a infecção por Gramnegativos é muito freqüente. Alguns dos Gram-positivos causadores de infecções, em pacientes neutropênicos, podem ser resistentes à oxacilina (estafilococos coagulase-negativos, enterococos resistentes a vancomicina, ou Corynebacterium jeikeium), porém, como a infecção por tais germes é mais indolente, o início da antibioticoterapia com vancomicina pode ser postergado alguns dias, sem aumento do risco de vida, embora se prolongue a duração da internação. Embora infecções fúngicas sejam, geralmente, superinfecções, também podem ser primárias

\section{5- ESCOLHA DO ANTIBIÓTICO}

A antibioticoterapia empírica deve ser iniciada prontamente, instituindo-se tratamento com drogas bactericidas, de largo espectro, por via endovenosa e em doses terapêuticas máximas. Os pacientes devem ser monitorados com freqüência (no mínimo duas vezes ao dia, se estáveis) quanto à resposta clínica, aparecimento de infecções secundárias, efeitos adversos, e surgimento de infecção por germes resistentes. A Figura 1 mostra o fluxograma proposto pela Infectious Diseases Society of America (IDSA). 


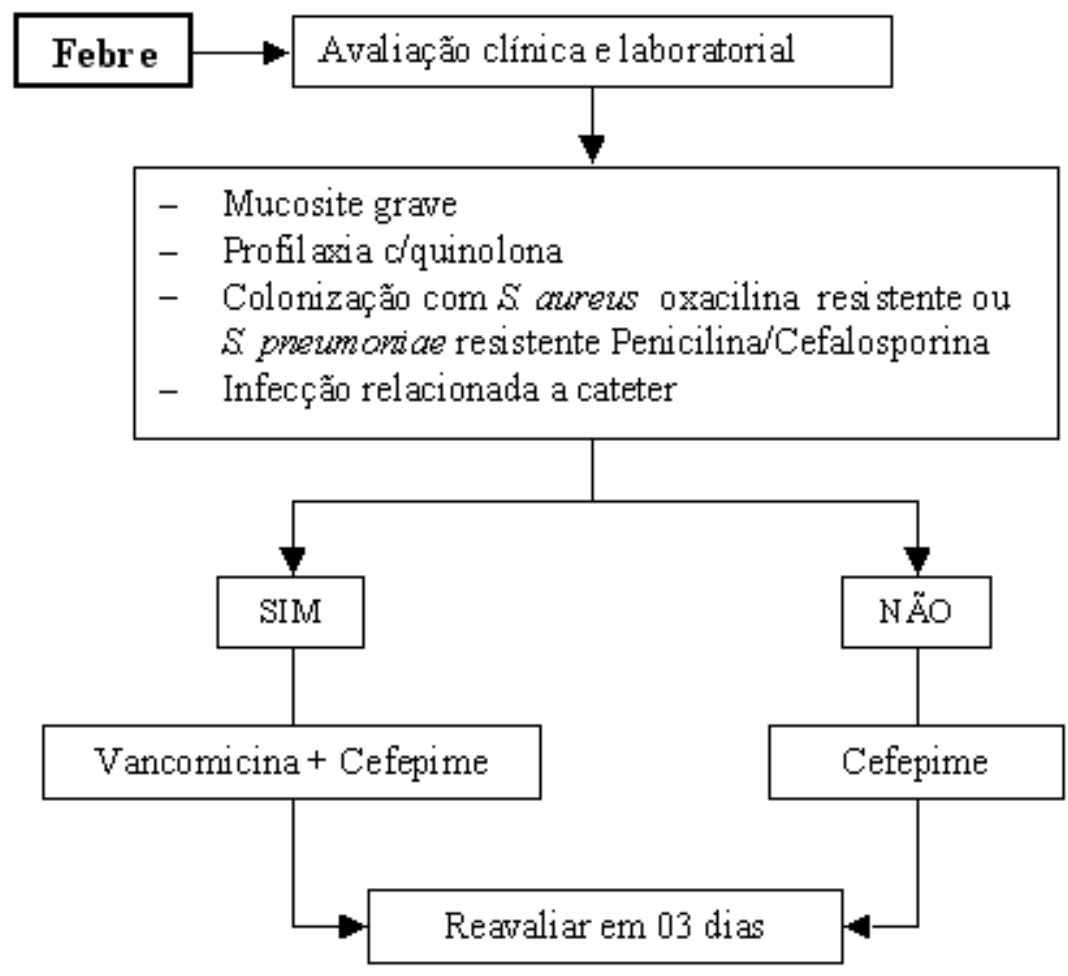

Figura 1: Fluxograma proposto pela Infectious Diseases Society of America (IDSA).

Estudos têm mostrado não haver diferenças entre monoterapia e combinações de duas drogas. Em nosso serviço, quando não há indicação de vancomicina, adotamos como monoterapia uma cefalosporina de $4^{\mathrm{a}}$ geração, cefepime, $2 \mathrm{~g} \mathrm{IV} \mathrm{12/12h} \mathrm{(caso} \mathrm{sejam} \mathrm{isola-}$ dos Pseu-domonas aeruginosa, enterobacter ou Klebsiella resistentes à amicacina ou ceftazidima, a dose deve ser aumentada para $2 \mathrm{~g}$ IV de $8 / 8 \mathrm{~h}$ ). Alternativamente, pode ser empregado um carbapenem

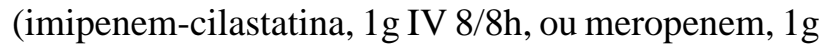
IV $8 / 8 \mathrm{~h}$ ), porém existe a preocupação com a emergência de cepas resistentes, e é preferível poupar o uso de tais drogas para infecções por agentes sabidamente sensíveis. As quinolonas e os aminoglicosídeos não devem ser usados como monoterapia inicial.

Vários serviços preconizam o uso da associação de um ß-lactâmico, anti-pseudomonas com um aminoglicosídeo. Os esquemas mais usados são: amicacina $(15 \mathrm{mg} / \mathrm{kg} / \mathrm{dia}$ dividida de $12 / 12 \mathrm{~h}$ ou 500 mg IV 12/12H diluídos em 100ml de soro fisiológico) com um dos três grupos de drogas: cefalosporina com atividade antipseudomonas (ceftazidima, cefepime), carboxi ou ureidopenicilina com atividade antipseudomonas (ticarcilina-clavulanato ou piperacilina-tazobactan) ou um carbapenem (imipenem-cilastatina ou meropenem). A literatura tem mostrado resultados semelhantes com as combinações acima, mas o esquema mais usado é ceftazidima + amicacina. O nível sérico dos aminoglicosídeos deverá ser monitorado, se possível, durante o tratamento. Há estudos em evolução com novas quinolonas (gatifloxacin, moxifloxacin e levofloxacin) em associação a $\beta$ lactâmicos ou glicopeptídeos (vancomicina).

As vantagens dos esquemas com duas drogas são o potencial efeito sinérgico e mínima emergência de resistência durante o tratamento, e as desvantagens são o aumento da toxicidade (nefrológica, otológica e alterações eletrolíticas como hipocalemia) e a não cobertura para alguns Gram-positivos, como $S$. aureus oxacilina sensível, $S$. oxidases e pneumoco$\cos$ (exceto com cefepime e ticarcilina-clavulanato).

A associação de vancomicina ao esquema empírico inicial só deve ser considerada em situações especiais, como: 
a) infecções graves, relacionadas a cateter (ex.: bacteriemia, celulite),

b) colonização conhecida do paciente por pneumococo resistente à penicilina e cefalosporina ou $S$. aureus resiste à oxacilina,

c) hemocultura positiva para Gram-positivo (ainda não identificado),

d) instabilidade cardiovascular,

e) mucosite grave pós-quimioterapia em altas doses,

f) pacientes em uso profilático de quinolonas.

A dose preconizada da vancomicina é de $1 \mathrm{~g}$ IV 12/12h, diluído em 100ml de soro fisiológico, em $1 \mathrm{~h}$.

Havendo suspeita de infecção por anaeróbios, tais como espécies de Clostridium não perfringens, associadas à gengivite necrotizante, celulite perianal, deve-se associar outro antibiótico, como metronidazol (500 mg IV 6/6h ou 8/8h) ou clindamicina (600 mg IV $8 / 8 \mathrm{~h}$ ou $12 / 12 \mathrm{~h})$.

\section{6- O PACIENTE COM CATETER VENOSO CENTRAL}

Os principais agentes de infecções relacionadas com cateter venoso central são Staphylococcus aureus e Estafilococo coagulase-negativo, e, geralmente, há resposta ao tratamento com antibiótico endovenoso, sem que haja retirada do cateter. É controversa a tentativa de, além do tratamento sistêmico, "tratar" o cateter com infusão de antibióticos pelo lúmen do cateter ou com soluções contendo antibiótico e heparina (antibiotic lock therapy).

Caso haja tunelite, embolia séptica e hipotensão associada à suspeita de infecção relacionada ao cateter, o mesmo deve ser retirado imediatamente. Define-se tunelite como flogose ao longo do trajeto subcutâneo de cateter implantado, em mais que $2 \mathrm{~cm}$ do local de saída do mesmo, com ou sem infecção concomitante da corrente sanguínea. Outras indicações de retirada imediata do cateter são infecções por espécies de Bacillus ou Acinetobacter, Pseudomonas aeruginosa, Stenotrophomonas maltophilia, Corynebacterium jeikeium, enterococos resistentes a vancomicina e fungemia. O cateter deve ser retirado e feito debridamento do tecido infectado.

\section{7- MANEJO DA ANTIBIOTICOTERAPIA NA PRIMEIRA SEMANA DE TRATAMENTO}

A mudança no esquema de antibióticos dependerá do quadro clínico do paciente e do tempo espera- do para a recuperação do número de granulócitos no sangue periférico. A Figura 2 mostra o fluxograma proposto pelo guia Infectious Diseases Society of America (IDSA) para avaliação dos resultados do tratamento após $72 \mathrm{~h}$.

Caso o paciente esteja afebril após $72 \mathbf{h}$ de tratamento e não haja documentação clínica ou laboratorial de infecção, deve ser mantido o tratamento inicial em pacientes com neutrófilos abaixo de $100 / \mathrm{mm}^{3}$ (independentemente da melhora clínica) ou em pacientes com mais de $500 / \mathrm{mm}^{3}$, que tenham algum sinal de instabilidade hemodinâmica ou do quadro clínico. Deve ser mantida antibioticoterapia por, no mínimo, 07 dias ou até que culturas indiquem que a infecção foi erradicada e o paciente esteja assintomático e com neutrófilos acima de 500 células $/ \mathrm{mm}^{3}$ por, no mínimo, 02 dias consecutivos. Se em 72 horas o paciente apresentar-se clinicamente bem, tiver neutrófilos acima de 500 células $/ \mathrm{mm}^{3}$ por, no mínimo, 02 dias consecutivos, sem diarréia ou mucosite, com culturas negativas, pode ser substituído o esquema antibiótico anterior por Ciprofloxacin oral (500 mg 12/12 horas) até completar 07 dias.

Se o paciente estiver afebril após $72 \mathrm{~h}$ de tratamento e tenha sido isolado o agente causador da infecção, deve ser ajustado o esquema antibiótico, baseado nas culturas e antibiograma, mas, sempre, sendo mantida a cobertura para germe Gram-negativo, mesmo que tenha sido isolado Gram-positivo. A antibioticoterapia deverá ser mantida por, no mínimo, 07 dias, ou até que não haja evidência microbiológica de infecção e o paciente esteja assintomático e com recuperação da medula óssea (neutrófilos acima de 500 células $/ \mathrm{mm}^{3}$ ). As bacteremias por $S$. aureus são tratadas por 14 dias, e por enterobactérias por 10 dias.

É importante ressaltar que caso não seja isolado, em culturas, germe que necessite do uso da Vancomicina para sua erradicação, a mesma pode ser retirada após $72 \mathrm{~h}$, se o paciente estiver estável clinicamente.

Nos casos em que o paciente não apresente previsão de recuperar a contagem de neutrófilos (como em anemias aplásticas e síndromes mielodisplásicas), se o paciente estiver clinicamente bem, sem sinais de infecção, com culturas negativas e sem fatores de alto risco (como neutrófilos abaixo de 100 células $/ \mathrm{mm}^{3}$ ou mucosite), pode ser interrompido o tratamento endovenoso após 05 a 07 dias completos sem febre, geralmente, fornecendo-se antibiótico oral para casa 


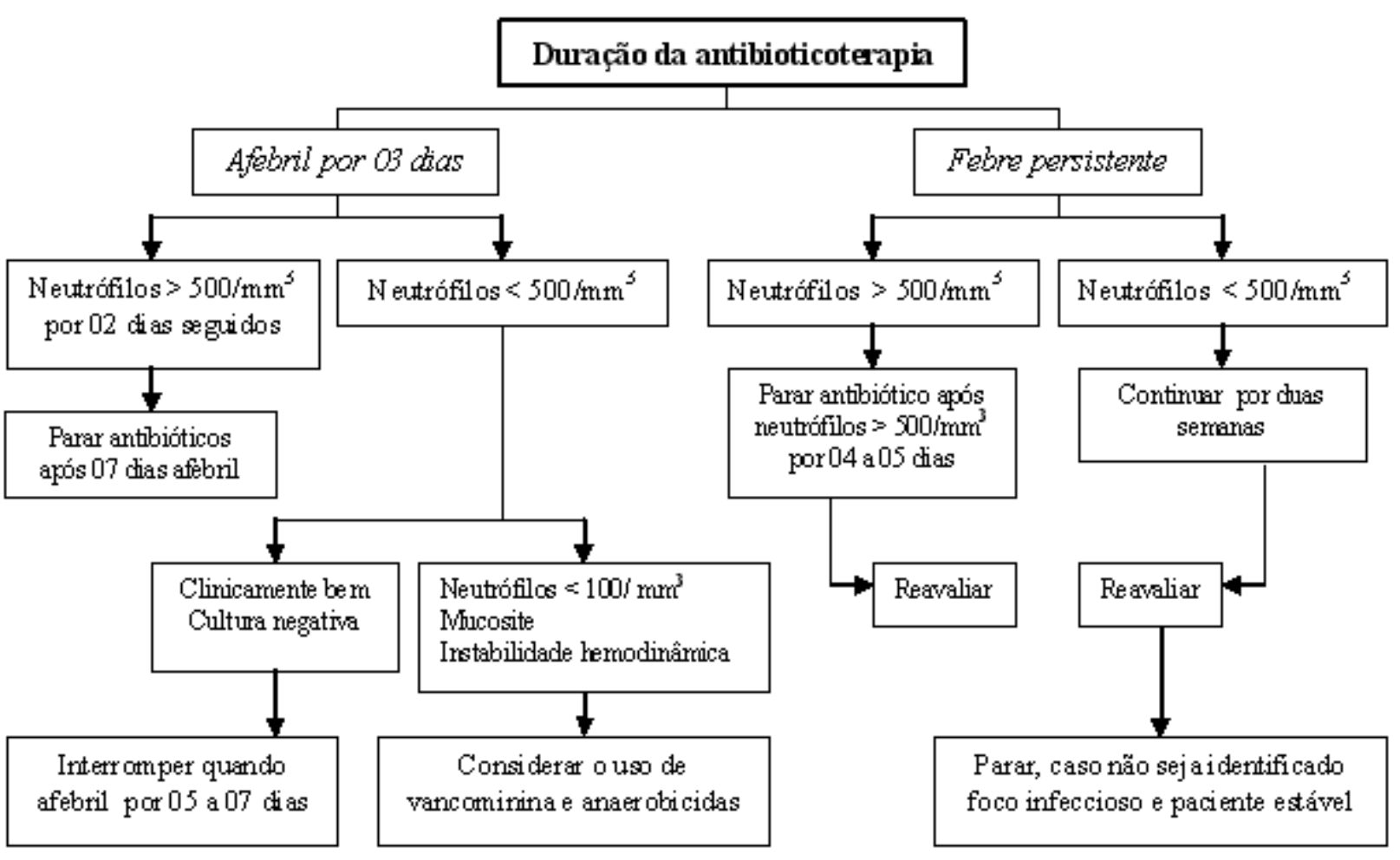

Figura 2: Fluxograma proposto pelo guia Infectious Diseases Society of America (IDSA)

(amoxicilina-clavulanato ou cefuroxima, preferencialmente o primeiro antibiótico).

Se o paciente estiver febril após $\mathbf{7 2}$ h de tratamento e sem documentação clínica ou laboratorial de infecção, deve ser mantido o esquema inicial, caso o mesmo esteja estável. Se o paciente apresentar piora ou qualquer sinal de instabilidade, pode ser associada a Vancomicina.

Se o paciente estiver febril após $\mathbf{7 2}$ h de tratamento e com documentação clínica ou laboratorial de infecção, deve-se adequar o antibiótico com os resultados das culturas, mantendo-se a cobertura para Gram-negativos.

Caso o paciente esteja febril após 05 dias completos de tratamento, e sem documentação clínica ou laboratorial de infecção, devem ser investigadas outras causas de febre, como, por exemplo, infecção fúngica, reações a drogas, febre pela própria doença de base, etc. Caso o paciente tenha radiografia de tórax normal, deve ser realizada CT de tórax de alta resolução. Caso o paciente tenha previsão de recuperar a contagem de neutrófilos para acima de
500 células $/ \mathrm{mm}^{3}$, nos próximos cinco dias, e esteja estável, pode ser simplesmente mantido o esquema inicial. Entretanto, estudos mostram que mais de um terço dos pacientes neutropênicos, febris, que não responderam a esquema de antibiótico por 01 semana ou mais, apresentavam, na maioria dos casos, infecção por Candida ou Aspergillus. Caso o paciente não esteja usando ainda, e principalmente se o mesmo for se manter neutropênico por um tempo mais longo (05 a 07 dias), pode ser iniciada anfotericina $\mathrm{B}$ até a dose de $1 \mathrm{mg} / \mathrm{kg} /$ dia (inicia-se por um dia com $0,5 \mathrm{mg} / \mathrm{kg} / \mathrm{dia}$, diluído em lipofundin 10\%, $100 \mathrm{ml}$, em 04 a 06 horas).

A duração do esquema de Anfotericina B é variável. Se houver documentação de infecção fúngica sistêmica, a duração vai ser determinada pelo tipo do agente e extensão da lesão. Se não houver confirmação da infecção, há controvérsias. Em geral, sem documentação de infecção fúngica e quando a neutropenia estiver resolvida, CTs de tórax e abdome estiverem normais, e o paciente estiver clinicamente bem, a medicação pode ser suspensa. Para pacientes que estão bem, sem infecção fúngica documentada, 
com neutropenia prolongada, sem lesões identificadas ao exame físico, radiografias ou tomografias de tórax, tomografias de abdome e em uso de mais de duas semanas de Anfotericina B, seu uso pode ser suspenso. Para pacientes de alto risco ou com estado geral ruim, deve ser continuado o tratamento até a recuperação da medula óssea.

Pacientes que se mantenham febris após a recuperação do número de granulócitos em sangue periférico (mais de 500 células $/ \mathbf{m m}^{3}$ ) deveriam ser reavaliados quanto a infecções fúngicas (principalmente candidíase sistêmica, aspergilose, histoplasmose, tricosporonose), por micobactérias ou vírus.

\section{8- USO DE DROGAS ANTIVIRAIS}

Não deve ser iniciado empiricamente, só quando há lesões identificadas. Infecções sistêmicas por citomegalovírus (CMV) não são causas comuns de febre nos pacientes neutropênicos febris, exceto naqueles submetidos a transplantes de medula óssea. Como as lesões orais dos neutropênicos não permitem fazer diagnóstico etiológico pelo seu aspecto, deve ser colhida citologia para coloração de Schorr, com o objetivo de identificar inclusões virais. Caso seja indicado tratamento, o uso de aciclovir $(10 \mathrm{mg} /$ $\mathrm{kg} /$ dia IV 8/8h) deve ser iniciado para Herpes simples ( podem ser usados ainda valaciclovir ou famciclovir, se disponíveis). Para CMV, indica-se ganciclovir ou foscarnet. A dose preconizada de ganciclovir é de 5 $\mathrm{mg} / \mathrm{kg}$ diluídos em $100 \mathrm{ml}$ de SG5\%, IV, em 1 h, de 12/ $12 \mathrm{~h}$, por 14 dias. A seguir deve ser feita a dose de 6 $\mathrm{mg} / \mathrm{kg} /$ dia diluídos em $100 \mathrm{ml}$ de SG5\%, IV, em 1h, uma vez ao dia, 05 vezes por semana, por tempo inde- finido. A dose de Foscarnet é de $60 \mathrm{mg} / \mathrm{kg} \mathrm{IV}$, em $1 \mathrm{~h}$, de $8 / 8 \mathrm{~h}$, por 14 a 21 dias.

\section{9- TRANSFUSÕES DE GRANULÓCITOS}

Não são feitas de rotina. Podem ser úteis para pacientes com neutropenia profunda, com infecção bacteriana ou fúngica, microbiologicamente documentada e que não esteja sendo controlada com antibiótico adequada ou com o uso de fator estimulador de colônia de granulócitos (G-CSF). As transfusões acarretam risco de transmissão de CMV, aloimunização associada com febre, reações enxerto versus hospedeiro (se os granulócitos não forem irradiados), refratariedade progressiva a plaquetas e possivelmente, insuficiência respiratória.

\section{0- USO DE FATORES ESTIMULADORES DE COLÔNIA}

O uso de fatores estimuladores de colônia de granulócitos (filgrastima ou G-CSF) ou de granulócitos e macrófagos (sarframostima ou GM-CSF) pode reduzir a duração da neutropenia, mas não altera a morbidade, a mortalidade e a duração da antibioticoterapia. Assim sendo, não deve ser de rotina, exceto quando houver expectativa de neutropenia prolongada ou perspectiva de piora do quadro clínico (pneumonia, celulite ou sinusite grave, hipotensão, infecções fúngicas sistêmicas e disfunção de múltiplos órgãos, secundária a sepse) ou, ainda, quando o paciente mantém-se gravemente neutropênico com infecções documentadas, que não respondem ao tratamento.

SOUZA DLCF \& REGO EM. Treatment of infections in patients with pancytopenia resulting from cytoreductive treatment. Medicina, Ribeirão Preto, 36: 446-452, apr./dec. 2003.

ABSTRACT - Fever of unknown origin is the most frequent cause of hospitalization of patients in treatment with cytoreductive regimens for neoplastic diseases. Patients present with no or unspecific symptoms, and the initial evaluation requires detailed history and physical examination, together with laboratorial exams aiming to identify the site of infection and complications secondary to chemotherapy. Treatment with broad spectrum antibiotics should be started immediately, and the therapy should be reevaluated after 72 hours based on the results from blood fluids cultures. In this review, we will discuss about the diagnostic and therapeutic approaches in febrile neutropenia.

UNITERMS - Neutropenia; diagnosis. Antibiotics. Fever. Pancytopenia. 


\section{BIBLIOGRAFIA RECOMENDADA}

1 - HUGHES WT; ARMSTRONG D; BODEY GP; BOW EJ; BROWN AE; CALANDRA T; FELD R; PIZZO PA; ROLSTON KVI; SHENEP JL \& YOUNG LS. Guidelines for the use of antimicrobial agents in neutropenic patients with cancer. Clin Infect Dis 34: 730751, 2002.

2 - MERMEL LA; FARR BM; SHERERTZ RJ; RAAD II; O'GRADY N; HARRIS JS \& CRAVEN DE. Guidelines for the management of intravascular catheter-related infections. Infect Control Hosp Epidemiol 22: 222-242, 2001.
3 - NUCCI M. Infecções no paciente com neoplasia hematológica: Diagnóstico, tratamento e prevenção. In: ZAGO MA; FALCÃO RP \& PASQUINI R. Hematologia: fundamentos e prática. Atheneu, São Paulo, cap. 41, p.. 419-429, 2001.

4 - FREEDENBERG WR. Disorders of granulocytes: qualitative and quantitative. In: MAZZA JJ. Manual of clinical hematology. 3th. ed. Lippincott, Williams and Wilkins, Philadelphia, cap. 7, p. 155-179, 2002. 